\title{
Irradiation of carbon nanotubes with carbon projectiles. A molecular dynamics study
}

\author{
Cristian D. Denton ${ }^{*, 1}$, Santiago Heredia-Avalos ${ }^{* *, 2}$, and Juan Carlos Moreno-Marín ${ }^{2}$ \\ ${ }^{1}$ Departamento de Física Aplicada, Universidad de Alicante, E-03080, Spain \\ ${ }^{2}$ Departamento de Física, Ingeniería de Sistemas y Teoría de la Señal, Universidad de Alicante, E-03080, Spain
}

Received ZZZ, revised ZZZ, accepted ZZZ

Published online ZZZ (Dates will be provided by the publisher.)

Keywords (Carbon nanotubes, Ion irradiation, Defect annealing, Computer Simulations)

* Corresponding author: e-mail denton@ua.es, Phone: +34 965903 400, Fax: +34 965909726

** e-mail sheredia@ua.es, Phone: +34 965903 400, Fax: +34 965909750

\begin{abstract}
The irradiation of carbon based nanostructures with ions and electrons has been shown to be an appropriate tool to tailor their properties. The defects induced in the nanostructures during irradiation are able to modify their mechanical and electronic properties. Here we simulate the irradiation of carbon nanotubes with carbon ions using a molecular dynamics code. We use the Tersoff potential joined smoothly to the Universal Ziegler-Biersack-
\end{abstract}

1 Introduction Since their discovery in 1991 [1] carbon nanotubes (CNTs) have attracted a lot of attention due to their outstanding electronic and mechanical properties [2]. It has been shown that CNTs can be metals or narrow band semiconductors depending on their chirality. These properties, in addition to the fact that they have a nanometer size, make them ideal candidates for use in nanoelectronics [3]. CNTs have also extremely high Young modulus and strength, which are expected to be, respectively, about 19 and 56 times that of steel [4]. As a consequence of these excellent mechanical properties CNTs can be used for reinforcements of other materials [2,5]. Many other exciting applications for CNTs have emerged in the last years [2].

In order to use CNTs in these potential applications, it is necessary to develop effective methods to tailor their properties. A possible solution is the use of energetic particles such as electrons, protons or ions [6]. Irradiation of CNTs with particles can implant foreign atoms, create defects, produce amorphous regions or recristallize the lattice. This technique is specially suited for manipulation of CNTs since the graphitic structure has a tendency to recon-

\begin{abstract}
Littmark potential at short distances. We study the number of defects produced after irradiation with a single carbon ion finding a saturation with its energy at $\sim 3$ $\mathrm{keV}$. We observe, after continuum irradiation with low energy ions, the formation of bumps in the irradiated region. For larger energy ions we find that the diameter of the nanotube shrinks as shown in previous works.
\end{abstract}

Copyright line will be provided by the publisher

struct the point defects by saturating dangling bonds [7]. The irradiation with ion or electron beams can also be used to coalesce [8], weld [9], link [10] or cut [11] the CNTs. This could allow the creation of molecular junctions and multiterminal heterostructures in future carbon-based nanoelectronics.

These technological applications need a thorough knowledge of the irradiation effects in the electrical and mechanical properties of CNT after irradiation. Thus it is important to analyze the mechanisms of production of defects under irradiation and also the changes in the structure induced by irradiation due to the saturation of the dangling bonds. Several papers have been devoted, both theoretically and experimentally, to the study of the effects of the ionic irradiation in CNTs and related nanostructures [6-7, 12-16].

In this paper we study the production of defects after irradiation of CNTs with carbon ions, using molecular dynamics simulations. We analyze the changes in the irradiated CNTs for different energies of the incident ion and dose. 
2 Simulation details We have developed a code based on classical molecular dynamics (MD) in order to simulate the bombardment of CNTs by carbon projectiles. Using this simulation code we have studied the defects produced on CNTs by carbon projectiles with incident energies from $10 \mathrm{eV}$ to $4 \mathrm{keV}$.

Because of the low energies involved in this work, we have neglected the electronic stopping, being the collisional process governed by the nuclear interactions.

In order to describe the C-C interactions, we have used the Tersoff potential [17], smoothly linked to the ZieglerBiersack-Littmark (ZBL) universal potential at short interatomic distances [18] to describe realistically the hard collisions between energetic atoms. Therefore, the interatomic potential was evaluated according to:

$$
V(r)=V_{\mathrm{ZBL}}(r)[1-F(r)]+V_{\text {Tersoff }}(r) F(r)
$$

where $F(r)$ is a Fermi function:

$$
F(r)=\frac{1}{1+\exp \left[b_{\mathrm{f}}\left(r-r_{\mathrm{f}}\right)\right]}
$$

being $b_{\mathrm{f}}=14 \AA^{-1}$ and $r_{\mathrm{f}}=0.95 \AA$. This potencial has already been used to study the ionic irradiation of graphite [12], as well as the B and N implantation in CNTs [15].

We have solved the equations of motion of all interacting atoms using the velocity Verlet algorithm [19] with a variable time step, which is stated considering that the maximum distance the atoms can move between two consecutive time steps. This distance was chosen checking that our simulation code satisfies the law of conservation of energy. It is worth to mention that, in order to avoid the displacement of the CNT when bombarding with carbon projectiles, we have kept fixed the atoms at both ends of the CNT during the simulation.

On the other hand, we have used the Berendsen thermostat to control the temperature of the CNT [20]. In this scheme, we have scaled the velocity of some atoms, placed near the CNT ends, according to the scaling factor:

$$
\lambda=\sqrt{1+\frac{\Delta t}{\tau}\left(\frac{T_{0}}{T}-1\right)}
$$

where $\Delta t$ is the time step of the simulation, $\tau$ is the time constant to control the bath coupling, $T_{0}$ is the desired temperature, and $T$ is the instantaneous temperature.

3 Results and discussion We have considered in our simulations single-walled carbon nanotubes (SWCNT) armchair $(10,10), \sim 100 \AA$ in length and $\sim 14 \AA$ in diameter; both ends have fixed positions, whereas the velocity of the carbon atoms corresponding to $5 \%$ of the total length of the CNT next to its fixed ends are scaled according to the Berendsen thermostat, as described previously.

It is worth to mention that the carbon projectiles bombard the CNT in the direction perpendicular to the nanotube axis, although the exact impact coordinates are ran- domly distributed on a central region of $10 \%$ of the total length of the CNT, as shown in figure 1.

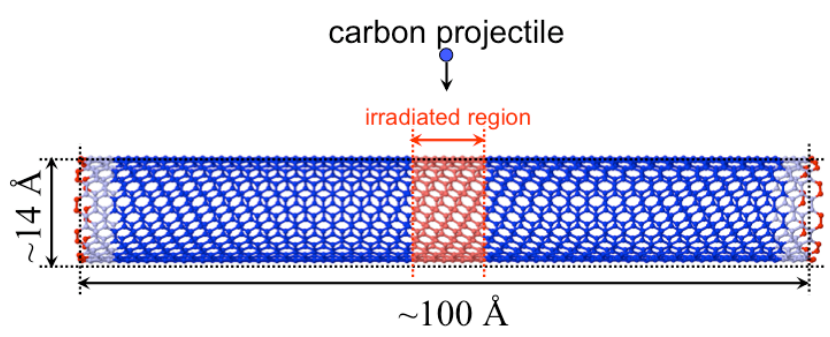

Figure 1 SWCNT with armchair $(10,10)$ structure, $\sim 100 \AA$ in length and $\sim 14 \AA$ in diameter. Red atoms at both ends of the CNTs have fixed positions during simulations, whereas Berendsen thermostat was applied to light blue atoms next to the CNT ends. Carbon projectiles bombard the CNT centre in the direction perpendicular to the CNT axis and along $10 \%$ of the CNT length.

We have divided the carbon irradiation of CNTs in three stages. The first one reproduces the impact of the projectile in the direction perpendicular to the CNT axis. We simulate this stage during $20 \mathrm{ps}$ and the temperature of the Berendsen thermostat is stated at $300 \mathrm{~K}$. The second stage is the posterior heating of the CNT during $50 \mathrm{ps}$, being $3000 \mathrm{~K}$ the temperature of the Berendsen thermostat. Finally, at the third stage we cool the CNT to a temperature of $0 \mathrm{~K}$ during $15 \mathrm{ps}$. These stages can be observed on figure 2, where we have depicted the temperature of the CNT as a function of the simulation time after the irradiation with a $200 \mathrm{eV}$ carbon projectile at $0.5 \mathrm{ps}$.

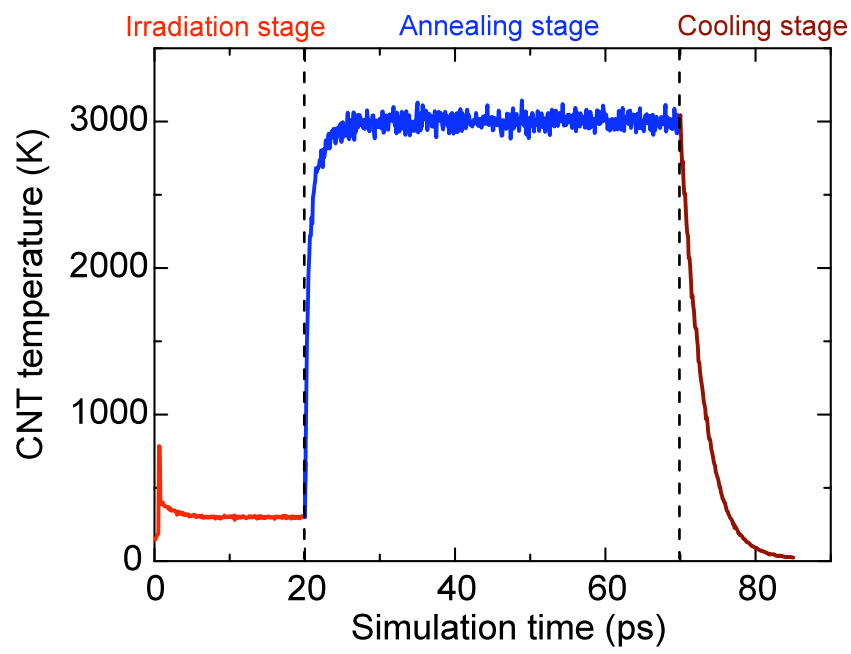

Figure 2 CNT temperature as a function of simulation time after the irradiation with a $200 \mathrm{eV}$ carbon projectile at 0.5 ps. The curves represent the CNT temperature during: irradiation stage (red), annealing stage (blue), and cooling stage (brown).

When carbon ions hit the CNTs wall, the main damage mechanism is the transfer of energy that results in the dis- 
placement of the atoms of the structure, and hence, the vacancies generation. Furthermore, depending on the transferred energy, the primary atoms can be backscattered, they can replace or extract atoms of the CNT, or even they can be added to the CNT (adatoms).

We have studied two different situations in our simulations. Firstly, we have evaluated the damage produced in the CNT due to individual carbon ion bombardment; secondly we have also analyzed the damage caused by consecutive carbon ions bombardment.

3.1 Single projectile irradiation Single-ion bombardment of the CNT causes damage, which can be evaluated through the formation of defects in the structure. According to previous authors [14], we assume that the coordination defect number reflects quantitatively the irradiation damage produced. This magnitude is defined as the number of carbon atoms with coordination number different than three.

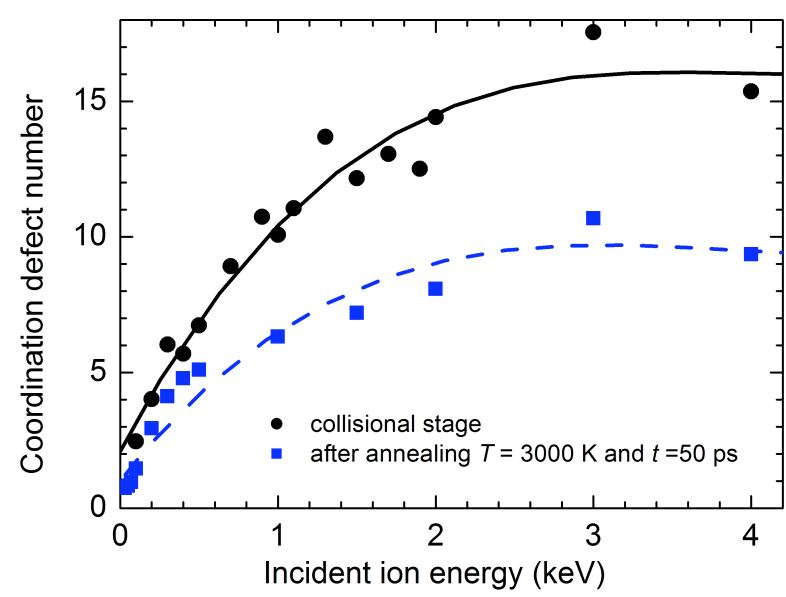

Figure 3 Number of coordination defects as a function of the incident projectile energy. After the collisional stage (black circles) and after the subsequent annealing stage (blue squares). The curves were depicted to guide the eye.

In figure 3 we present the results of the coordination defect number obtained after the irradiation with carbon ions of different incident energies (black circles) and its evolution after the annealing stage of the CNT (blue squares); the curves were depicted to guide the eye. We can see that the coordination defect number increases with incident ion energy up to $3 \mathrm{keV}$, reaching a saturation value $\sim 15$ at higher energies. In addition, figure 3 shows that after the irradiation stage, the heating of the CNT in the annealing stage helps the migration of interstitial atoms and vacancies and their recombination, so that the coordination defect number decreases.

The irradiation of low energy carbon ions causes the adhesion of carbon atoms to the CNT wall (single adatoms), which evolved after annealing to the formation of double-coordinated atoms (bridge defects). Figure 4(a) shows a typical transformation of an adatom to a bridge defect after annealing, when a $10 \mathrm{eV}$ carbon projectile hits on the CNT during the irradiation stage. However, for higher projectile energies, the irradiation also causes the formation of vacancies, resulting in different size holes that can evolve after the annealing stage to non-hexagonal structures, called Stone-Wales defects. We show in figure 4(b) the typical transformation of two vacancies into a Stone-Wales defect after annealing; in this case the projectile hits the CNT with $100 \mathrm{eV}$. In both figures the colours indicate the coordination number: white (1), red (2), blue (3), and green (4).

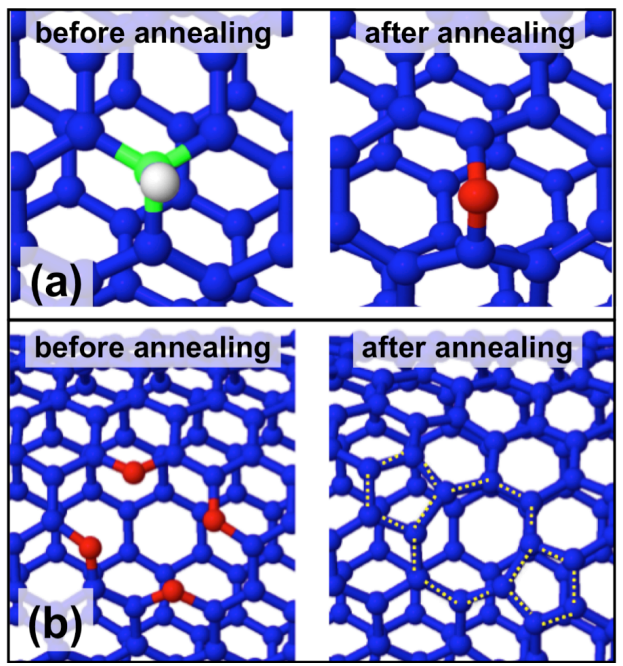

Figure 4 Typical transformation of defects during annealing after irradiation with: (a) $10 \mathrm{eV}$ and (b) $100 \mathrm{eV}$ carbon ion. Colours mean the coordination number: white (1), red (2), blue (3), green (4).

3.2 Continuum projectile irradiation On the other hand, we have studied the damage produced on the CNT when it is successively irradiated with carbon ions.

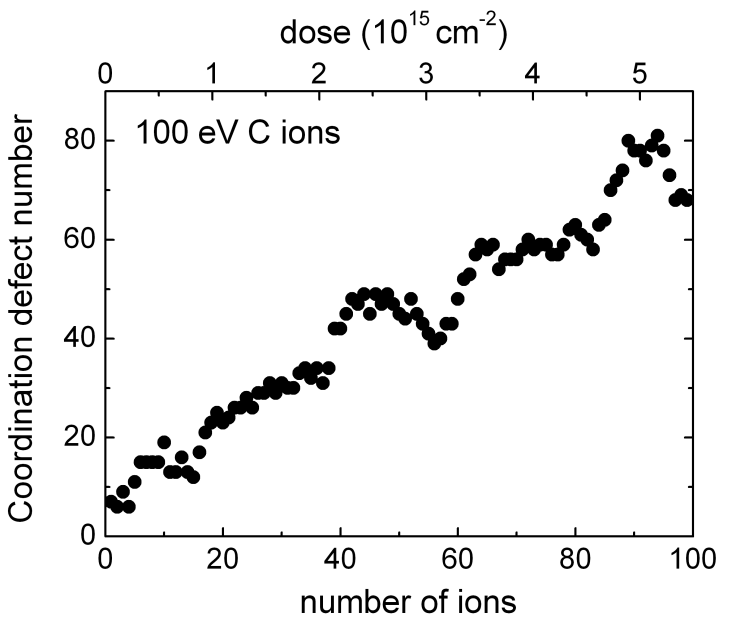

Figure 5 Coordination defect number as a function of the number of ions (ion dose) that bombard the CNT with $100 \mathrm{eV}$ energy. 
We have depicted in figure 5 the coordination defect number as a function of the number of incident carbon ions; the energy of all carbon projectiles is $100 \mathrm{eV}$. As expected, we have found in our simulations that the coordination defect number grows proportionally to the incident ionic dose. In addition, we have observed that the coordination defect number increases with the projectile energy.

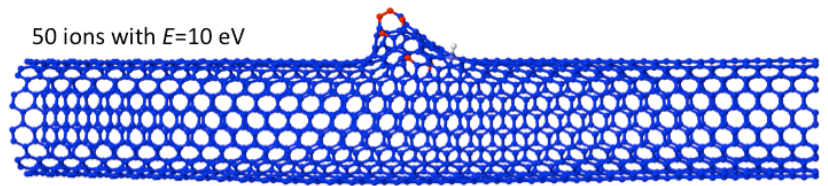

50 ions with $E=100 \mathrm{eV}$

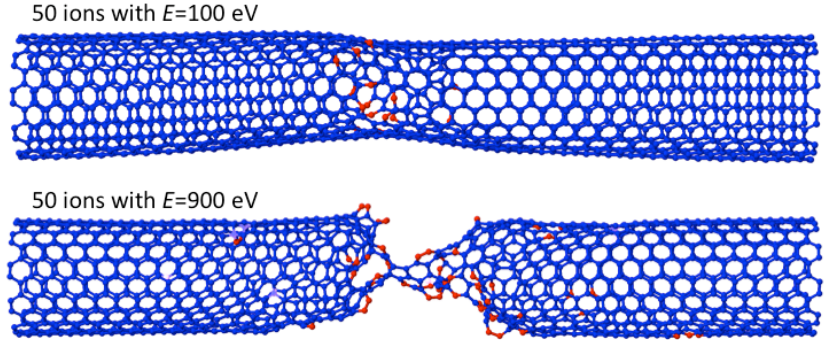

Figure 6 Appearance of CNT after irradiation with 50 consecutive carbon ions with different energies.

The effects of the continuous irradiation of the CNT, depending on the energy of the incident projectile, are shown in figure 6 , in which we present the appearance of the CNT after irradiation with 50 projectiles; colours represent the coordination number of the carbon atoms as previously stated.

With low energy ions $(\sim 10 \mathrm{eV})$, adatoms are produced in the walls of the CNT, which can evolve into the formation of bumps on the irradiated region, as observed in figure 6. When the projectiles have higher energies (100-1000 $\mathrm{eV})$, vacancies appear during the irradiation stage, which evolve, during the annealing, into polygonal shapes. In addition, a progressive reduction of the CNT diameter is observed, as shown in figure 6 . Increasing the dose results in a higher damage, which could even break the CNT in the irradiated area.

4 Conclusions We have developed a MD code in order to simulate the irradiation of nanostructures. In this work, we use this code to study the irradiation of CNTs with carbon ions.

We have evaluated the damage produced by individual ions in a perfect CNT. We find that for low projectile energies $(E<50 \mathrm{eV})$ the defects are only adatoms, while vacancies or cluster of vacancies are also observed for higher energies. These defects are partly healed after annealing through the saturation of dangling bonds. The number of defects generated by a single ion grows with projectile energy but saturates at $\sim 3 \mathrm{keV}$.

We have also studied the effect of continuum irradiation of the CNT. For low energies the accumulation of adatoms produces a bump in the irradiated region. At intermediate energies the irradiation produces vacancies which are healed through Stone-Wales defects. This gives rise to a shrinking of the CNT diameter in the irradiated region which has been observed before [13]. If the projectile energy is high enough the continuum irradiation produces the breaking of the CNT.

Acknowledgements This work has been financially supported by Project FIS2010-17225 by the Spanish Ministerio de Ciencia e Innovación. The authors would like to thank M. J. Caturla for her help and suggestions.

\section{References}

[1] S. IIjima, Nature 354, 56 (1991).

[2] M. S. Dresselhaus. G. Dresselhaus, P. Avouris (Eds.), Carbon nanotubes, Synthesis, Structure, Properties and Applications (Springer, Berlin, 2001).

[3] P. L. Mc Euen, Nature 393, 15 (1998).

[4] R. H. Baughman, A. A. Zakhidov, W. A. de Heer, Science 297, 787 (2002).

[5] A. B. Dalton, S. Collins, E. MuÃ \pm oz, J. M. Razal, V. H. Ebron, J. P. Ferraris, J. N. Coleman, B. G. Kim, R. H. Baughman, Nature 703, 423 (2003).

[6] A. V. Krasheninnikov and K. Nordlund, J. Appl. Phys. 107, 071301 (2010)

[7] A. V. Krasheninnikov, F. Banhart, Nat. Mater. 6, 723 (2007).

[8] M. Terrones, H. Terrones, F. Banhart, J.-C. Charlier, P. M. Ajayan, Science 288, 1226 (2000).

[9] M. Terrones, F. Banhart, N. Grovert, J.-C. Charlier, H. Terrones, P. M. Ajayan, Phys. Rev. Lett. 89, 075505 (2002).

[10] H. Stahl. J. Appenzeller, R. Martel, P. Avouris, B. Lengeler, Phys. Rev. Lett. 85, 5186 (2000).

[11] M. S. Raghuveer, P. G. Ganesan, J. D'Arcy-Gall, G. Ramanath, M. Marshall, I. Petrov, Appl. Phys. Lett. 84, 4484 (2004).

[12] K. Nordlund, J. Keinonen, T. Mattila, Phys. Rev. Lett. 77, 699 (1996).

[13] A. V. Krasheninnikov, K. Nordlund and J. Keinonen, Phys. Rev. B 65, 165423 (2002).

[14] A. V. Krasheninnikov and K. Nordlund, Nucl. Instrum. Meth. Phys. Res. B 216, 355 (2004).

[15] J. Kotakoski, A. V. Krasheninnikov, Yuchen Ma, A. S. Foster, K. Nordlund, R. M. Nieminen, Phys. Rev. B 71, 205408 (2005).

[16] A. V. Krasheninnikov, J. Comp. Theor. Nanoscience 5, 1 (2008).

[17] J. Tersoff, Phys. Rev. B 39, 5566 (1989).

[18] J. F. Ziegler, J. P. Biersack and M. D. Ziegler, SRIM: The stopping and range of ions in matter (Lulu Press, Napa, 2008).

[19] M. P. Allen y D. J. Tildesley, Computer simulation of Liquids (Oxford Science Publications, Clarendon Press, Oxford, 2002).

[20] H. J. C. Berendsen, J. P. M. Postma, W. F. van Gunsteren, A. DiNola, and J. R. Haak, J. Chem. Phys. 81, 3684 (1984). 